\title{
Give the girls a chance: should spatial skills training be incorporated into the curriculum?
}

\author{
S. Jones \& G. Burnett \\ School of Computer Science and Information Technology, \\ The University of Nottingham, UK.
}

\begin{abstract}
Recently, tests for spatial reasoning skills were introduced in the UK for young children. However, effort is still directed at improving reading and mathematics, with no defined inclusion of spatial skills training in the curriculum. This is a cause for concern given that spatial skills are important for a range of ICT-related activities - for example, navigating Web sites. Furthermore, such abilities have implications for future subject and career choices. There are well-documented individual differences in spatial skills, with gender being one of the most studied. The current study aimed to determine if short-term training with online 2-D or 3-D spatial puzzles resulted in a greater increase in spatial skills compared to a control group trained with word puzzles. The study, involving 168 children between the ages of 6 and 11 years, investigated the impact of the dimensionality of puzzles, as well as gender, age and starting spatial score. Additionally, the children were questioned on the length of time they spent on computers, and their preferred computer-related pastimes. The results showed a trend for higher spatial scores after training with spatial puzzles compared to the control group, although these results did not reach statistical significance. Younger children demonstrated a greater increase than older children, this age trend being greater amongst the females. Those with lower starting spatial scores showed a significantly greater increase in spatial scores after training. As expected, males reported playing computer games more frequently than females. Furthermore, there was evidence that children with higher spatial scores were actively choosing to play computer games more than other ICT pastimes such as emailing. Based on these findings, a provisional recommendation is made that spatial skills training should be incorporated into the curriculum.
\end{abstract}

Keywords: spatial skills, gender differences, children, education. 


\section{Introduction}

Historically, children have undergone rigorous testing in the areas of verbal and numerical abilities, and recently tests for spatial reasoning skills were introduced in the UK for young children $[1,2]$. However, while schools concentrate on improving the skills of reading and mathematics, very little effort is put into the area of spatial reasoning within the curriculum [3, 4]. This is of some concern, especially as there is evidence of gender differences in spatial reasoning ability, with females appearing to under-perform in certain measures such as mental rotation $[5,6]$, these gender differences being detected in children as young as 4.5 years old [7]. Some researchers believe that mental rotation is a measure of a general spatial reasoning ability [8], and that this ability is a predictor of future subject choices (such as engineering) and careers (such as architecture and piloting aircraft) [2,9]. There is a growing recognition of the importance of testing for spatial ability, not just as a means for tailoring teaching methods, but also for improving these skills in the poor performers [2].

It is becoming accepted that a child's overall mental ability should no longer be assessed on verbal abilities alone [2, 3]. Halpern [3] states that verbal and visual-spatial abilities have been proven to be separate components of intelligence, and defines visual-spatial ability as a cognitive characteristic that gives a measure of a user's ability to conceptualise the spatial relationships between objects. Spatial abilities cover a broad area of cognition - Linn and Peterson [5] list three subgroups of spatial ability, Halpern [3] five, but both categorisations include the ability of mental rotation, which is the capacity to accurately picture the rotation of two or three dimensional objects in the mind. It is suggested that males and females use different strategies when carrying out a mental rotation test (MRT), with males using a more holistic or gestalt strategy, and females a slower, more serial part-by-part or verbal approach $[10,11]$. There is also evidence that females take longer to complete mental rotation tasks, perhaps because of the slower verbal strategy, but also because they are more cautious in their approach and spend more time confirming the correctness of their choices $[12,13]$.

There are various theories postulated for the gender differences in spatial abilities, with controversy surrounding the contribution of "nature" versus "nurture". One argument is the "bent twig" theory [14], whereby there is selfselection for choosing activities for which one is biologically pre-disposed (nature), thus contributing further to an increase in magnitude in those skills (nurture). If this is the case, the implication is that any genetically predisposed gender differences in spatial skills could be amplified by differential amounts of time spent on spatial tasks, and hence differential amounts of spatial training [6]. This would be thought to apply to anyone with poor spatial abilities, regardless of their gender. Studies have shown boys tend to pick more spatial toys such as Lego [15], spend more time playing computer games [16], and choose more spatial subjects such as mathematics, architecture and engineering [17]. It is now widely accepted that performance in spatial tests is positively impacted by informal participation in these types of spatial activities $[6,17]$. 
It has also been postulated that those with lower spatial scores should benefit more from spatial training by demonstrating a greater increase in spatial scores after the training period $[17,18]$. Various studies have demonstrated improvement in spatial ability measures in adults after intervention with spatial training techniques (for example, $[19,20]$ ). Few studies have looked at the impact of spatial training on spatial test performance in children. McClurg and Chaille [21] reported improvement in MRT scores of $57 \mathrm{fifth}$, seventh and ninth grade American pupils after training with computer games, again with both sexes showing equal improvement across the grades. Subrahmanyam and Greenfield [22], working with 61 American fifth grade students (10.5 to 11.5 year olds), demonstrated that higher initial spatial scores correlated with how the children performed in video games. Furthermore, limited training with video games (for three 45 minute sessions) resulted in an increase in spatial scores, especially in those with low starting scores.

The current study carried out a spatial training exercise with a larger number of young children in the UK, with a view to ascertaining the benefits of incorporating spatial training into the primary school curriculum. Gender, age, starting spatial score and puzzle dimension (2-D or 3-D) were incorporated as variables into the study. The objective was to determine the effects of these variables on any increase in spatial score after the training exercise, and to establish any relations between these variables and choice of computer activities.

\section{Method}

\subsection{Participants}

Participants consisted of 168 pupils from a primary school in Nottingham, UK. The pupils, comprising 81 girls and 87 boys between the ages of 6 and 11 years, were allocated to 3 groups with statistical equivalence on spatial scores, age and gender (see Table 1).

\subsection{Materials}

Individual differences in spatial skills were measured using the Spatial Reasoning tests devised by nferNelson [1]. The paper tests assess the child's ability to carry out a range of cognitive tasks concerning space, shape and mental rotation. The resultant raw score was converted to a nationally standardised score with the average being 100 . This allowed for comparison with children of the same age. The children were also required to complete a computer usage questionnaire to assess how much time they spend, and what activities they perform, on the computer.

Various on-line puzzles were supplied to the children for training purposes, consisting of mental rotation tasks (2-D and 3-D), maze completion tasks (2-D and 3-D), and word games (see figure 1 for examples). 


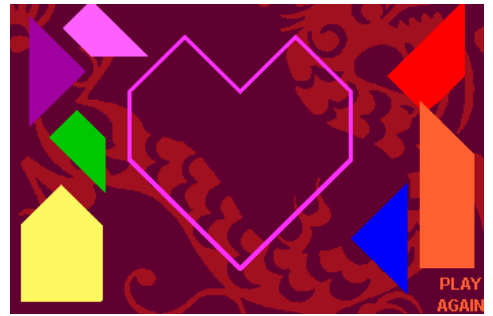

2-D rotation puzzle, source: www.kids.com

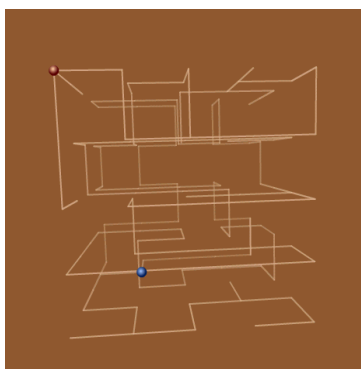

3-D maze, source: www.speedyadverts.com

Figure 1: $\quad$ Two of the online tests.

The 2-D rotation task consisted of the Chinese game of "Tangrams", where players are required to move, rotate or flip shapes to fit into an outline picture (source: www.kids.com, see figure 1). The 3-D rotation game utilised a rubric cube composed of small cubes rotated until each face of the rubric cube is one colour (source: www.aardasnails.com). The 2-D maze game was a standard maze tracing exercise (source: http://home.att.net/ jpunz/maze/maze.html), while the 3-D maze game allowed the player to rotate the whole maze structure (source www.speedyadverts.com, see figure 1). The word puzzles consisted of an online word search (source: http://www.dailywordsearch.com/) and a word matrix exercise (source: http://fun.familyeducation.com).

A facility for information technology classes was utilised on the school premises, consisting of 14 workstations running Windows 2000 and Internet Explorer version 6.

\subsection{Procedure}

The spatial ability test was administered to the children a week before the training sessions commenced, and the results used to randomly assign the children to three groups of equal age, sex and spatial score distributions. The computer questionnaire was administered at the same time. Once a week over the following three weeks, the children were exposed to a 20 minute training session, with group A working on the 2-D puzzles, group B on the 3-D puzzles, and group $\mathrm{C}$ acting as the control working with the word puzzles. Each group had a demonstration of the relevant puzzles with oral instructions prior to commencing the training, and were informed they would be awarded stars after reaching various points in the puzzles. They were not, however, informed about the purpose of the training, or its relation to the evaluation of spatial abilities. The children worked alone at each workstation, and the address bars were hidden to prevent them playing with the puzzles outside the training sessions. A week after the treatment ended, the spatial skills test was administered again. 


\subsection{Dependent variables}

Increase in spatial scores was the main parameter measured as a result of the intervention with spatial training.

\subsection{Independent variables}

\subsubsection{Training groups}

The groups were matched on gender split, age and starting spatial scores (starting spatial, $\mathrm{p}=0.99$ ).

Table 1: Training groups.

\begin{tabular}{ccccc}
\hline Group & Training & N (Female/Male) & Age $(\mathrm{yrs}) *$ & Starting spatial $^{*}$ \\
\hline A & 2-D spatial & $51(22 / 29)$ & $9.49(1.32)$ & $98.33(14.8)$ \\
B & 3-D spatial & $50(24 / 26)$ & $9.28(1.21)$ & $96.62(14.6)$ \\
C & Verbal & $44(22 / 22)$ & $9.29(1.31)$ & $95.16(14.05)$ \\
\hline
\end{tabular}

* Mean (S.D.s).

\subsubsection{Gender}

68 girls (mean age $=9.4 y e a r s$, S.D. $=1.41)$ and 77 boys (mean age $=9.3$, S.D. $=1.15$ ) were included in the analysis. Of these, $65 \%$ girls and $66 \%$ boys had access to a computer at home. $32 \%$ of girls and $36 \%$ boys rated themselves as high users of computers.

\subsubsection{Age/age category}

The children were assigned to two age categories. There were 82 children in the older age category $(9-11$ years, mean $=10.3$, S.D. $=0.76)$ and 63 in the younger age group (6-8years, mean=8.13, S.D. $=0.55)$.

\subsubsection{Original spatial/spatial category}

For ease of analysis, this continuous variable was divided into a 3-group categorical variable: high $(\mathrm{N}=48$, mean starting spatial=112.84, S.D. $=5.47)$, medium $(\mathrm{N}=53, \mathrm{M}=98.91$, S.D. $=4.14)$, and low $(\mathrm{N}=44, \mathrm{M}=79.71$, S.D. $=7.32)$.

\section{Results}

\subsection{Training groups}

A one-way between-groups analysis of variance (ANOVA) was conducted to explore the impact of spatial training on spatial scores. Although the results were not statistically significant $(\mathrm{F}(2,142)=0.75, \mathrm{p}=0.47)$, there is a trend for higher increase in spatial scores in the spatial training groups (group $\mathrm{A}, \mathrm{M}=5.45$, S.D. $=8.19$ and group $\mathrm{B}, \mathrm{M}=5.66$, S.D. $=8.12$ ) compared to the control group $\mathrm{C}$ $(\mathrm{M}=3.93$, S.D. $=5.16)$.

\subsection{Gender}

Although the boys recorded a higher mean original spatial score $(\mathrm{M}=97.69$, S.D. $=14.37)$ than the girls $(\mathrm{M}=95.75$, S.D. $=14.68)$, this difference was not 
statistically significant $(\mathrm{p}=0.42)$. Similarly, there was no significant difference $(\mathrm{p}=0.76)$ in the increase in spatial scores for the training groups between boys $(\mathrm{M}=5.78$, S.D. $=8.08)$ and girls $(\mathrm{M}=5.28$, S.D. $=8.25)$ over both age categories.

\subsection{Age category}

When gender was introduced as a factor, it was observed that the mean original spatial score was lower for younger girls $(\mathrm{M}=93.55$, S.D.=15.3) compared to older girls $(\mathrm{M}=97.38$, S.D.=14.19), and older $(\mathrm{M}=97.86$, S.D.=14.59) and younger boys $(\mathrm{M}=97.47$, S.D. $=14.31)$. This difference was not statistically significant $(\mathrm{p}=0.48)$.

An independent samples t-test was carried out to compare the increase in spatial scores for girls and boys in the different age categories in the training groups $(\mathrm{A}$ and $\mathrm{B})$. There was a significant difference $(\mathrm{t}=-2.32, \mathrm{p}<0.05)$ in mean increases for girls when comparing younger girls $(M=8.35$, S.D. $=8.33)$ with older girls $(\mathrm{M}=2.92$, S.D.=7.51). Younger and older boys showed comparable means $((M=6.35, S . D .=7.91)$ and $(M=5.38, S . D .=8.29)$ respectively $)$, and these did not differ significantly from the girl's results (see figure 2).

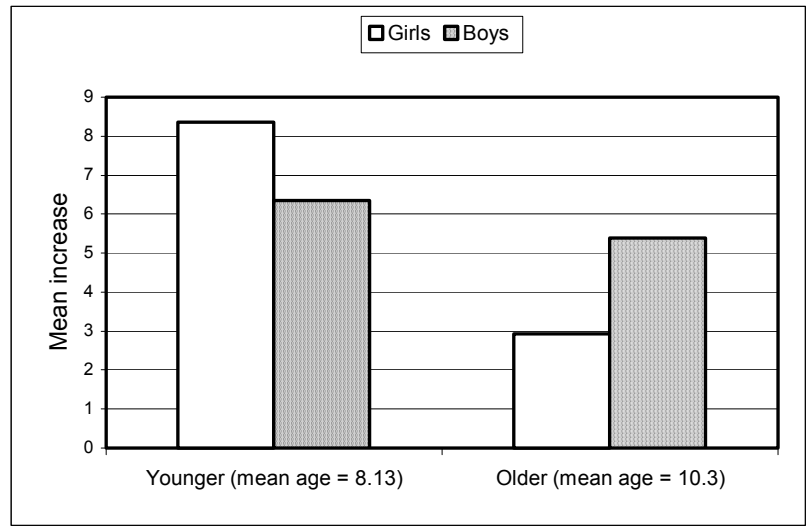

Figure 2: $\quad$ Mean increase in spatial scores by age and gender.

\subsection{Original spatial scores}

The continuous variable of original spatial scores was converted to a categorical variable consisting of high, medium and low spatial groups. A one-way betweengroups ANOVA was conducted to explore the impact of starting spatial score on the increase in post-training spatial score. There was a statistically significant difference for the increase in spatial scores between the three different original spatial scores groups $(\mathrm{F}(2,142)=11.3, \mathrm{p}<0.001)$. Post-hoc comparisons using the Tukey HSD test indicated that the mean increase in spatial score for the low spatial group $(M=8.79, S D=9.04)$ was significantly different at the $\mathrm{p}<0.001$ level from the medium $(\mathrm{M}=4.02, \mathrm{SD}=5.7)$ and high groups $(\mathrm{M}=2.25, \mathrm{SD}=5.3)$. This 
relationship between original spatial score and increase was significant in both genders (girls $-\mathrm{F}(2,65)=7.25, \mathrm{p}<0.01$; boys $-\mathrm{F}(2,74)=4.6, \mathrm{p}<0.05)$, and in the older children $(\mathrm{F}(2,79)=10.48, \mathrm{p}<0.001)$.

\subsection{Computer usage}

There was no relation between reported computer usage (obtained from the questionnaire) and either original spatial scores or increase in scores.

\subsection{Activities}

$56 \%$ of boys chose to play games as their primary choice of computer activity compared to $20 \%$ of girls. There was a trend for the mean starting spatial score of those who cited games as their primary computer pastime $(M=100.13$, S.D. $=14.83$ ) to be higher than those who preferred other tasks such as emailing and visiting chat rooms $(\mathrm{M}=96.96$, S.D.=13.94), although this did not reach significance $(\mathrm{p}=0.24)$.

\section{Discussion}

Although not statistically significant, there was a trend for a greater increase in spatial scores in the training groups compared to the control. The lack of significance may be explained by the fact that the training was carried out over three 20-minute sessions - Baenninger and Newcombe concluded from their meta-analysis that training should be at least of medium duration, defined as more than one administration over a course of more than three weeks [17]. However, De Lisi and Cammarano, working with Psychology students, recorded a statistical effect of training after only two 30-minute sessions [23]. Baenninger and Newcombe also found that the effectiveness of the training intervention was influenced by the similarity between the training and testing tasks [17]. In this study, the training tasks were not based on the tasks in the spatial tests, and both used different media (computer and paper); it would be interesting to repeat the procedure with an online version of the current spatial skills test. Another issue is that the children may have been subjected to varying amounts of spatial tasks outside the sessions and this could not be controlled for.

There were slight gender differences in the original spatial scores, and the subsequent increase in spatial scores, but these were not statistically significant. A possible reason is, while each section of the spatial reasoning test had time restrictions and most of the children finished within these limits, the observation was that boys tended to finish faster than the girls. As already mentioned, males seem to carry out mental rotation exercises faster than females, so it would be interesting to introduce an element of timing to completion of the tests to determine if this further differentiates the genders. Another argument is that, while recorded gender differences in the literature have been alluded to, there are inconsistencies in the findings, and some suggestion that the differences appear to be reducing over time [24]. In the Western world, young girls are increasingly exposed to spatial tasks - for example, female participation in football has 
increased in recent years [25] - and this could have an impact on the relative development of spatial skills.

Younger girls demonstrated a trend for lower starting spatial scores than the older girls, and the older and younger boys. This group also showed a significantly greater increase in spatial scores than the older girls, and a trend for a greater increase than the boys, resulting in a final spatial score very similar to the boys. This conforms with the expectation that those with lower starting spatial scores have a greater potential for increase. This assumption is reinforced by the significantly higher increase in the scores in the low spatial group compared to the medium and high groups when considered as a categorical variable. This result has implications for the preferred age to carry out the spatial training. While Subrahmanyam and Greenfield [22] found strong results with training of 10 year olds, and postulated this may be a sensitive age for spatial skill development, the largest increases seen in the current study were in the 6 to 8 year olds. McGuiness and Morley suggest some sort of spatial training should be commenced at nursery school level, thus pre-empting a female reliance on slower verbal strategies and allowing an early development of more spatial interests [26].

Voyer et al. [27] noted that most of the spatial testing materials employed in the studies included in their meta-analysis were designed for adults, and this would make it hard for the children to complete them. Kerns and Berenbaum [28] suggested that sex differences in children would only be demonstrated when utilising tests designed specifically for the test group. The spatial test utilised in this study was designed for children so as not to introduce the floor effect postulated by Voyer et al. [27]. However, the tool used was 2-D paper based, and this may explain why there was little benefit of the 3-D puzzles over the 2-D versions. As already stated, the training tasks were not based on the tasks used in the tests; however, it is difficult to ascertain how generalised the transfer effect was, and if it would affect performance levels in real life spatial tasks such as navigation or mathematics scores [4]. There was an increase in spatial scores in the control group who performed online word puzzles with no spatial element, suggesting a possible training impact associated with the test itself. However, no feedback was given as to the correctness of the children's answers during or after the tests.

The results of this study lend support to the environmental argument for some of the individual differences in spatial skills, with those with lower spatial abilities receiving the most benefit from the training intervention. There was evidence that those with higher spatial scores tended to pick more spatial computer activities; this would lend credence to the Bent Twig theory discussed earlier, where those with higher spatial abilities self-select for more spatial pastimes [6].

\section{Conclusion}

On the basis of this study, we can conclude that spatial training has the most potential for those with lower spatial scores, particularly the younger females, 
although it would be of value to all. As it would be relatively easy and inexpensive to incorporate these types of spatial training into the curriculum, there seems no justifiable argument against their inclusion. Future studies need to ascertain the required frequency and duration of training, determine how durable and generalisable the chosen training program may be, and investigate the benefits of the many different types of spatial training available.

\section{References}

[1] Smith, P. \& Lord, T.R., Spatial reasoning, a teacher's guide. nferNelson Publishing Company Ltd: London, UK, 2002.

[2] Smith, P., Spatial ability and its role in United Kingdom education. Vocational Aspect of Education, 44(1), pp. 103-106, 1992.

[3] Halpern, D.F., Sex differences in cognitive abilities. Lawrence Erlbaum Associates: Mahwah, New Jersey, 2000.

[4] Baenninger, M. \& Newcombe, N., Environmental input to the development of sex-related differences in spatial and mathematical ability. Learning and Individual Differences, 7(4), pp. 363-379, 1995.

[5] Linn, M.C. \& Peterson, A.C., Emergence and characterization of sex differences in spatial ability: a meta-analysis. Child development, 56, pp. 1479-1498, 1985.

[6] Voyer, D., Nolan, C., \& Voyer, S., The relation between experience and spatial performance in men and women. Sex Roles - A Journal of Research, 43(11/12), pp. 891-915, 2000.

[7] Levine, S.C., et al., Early sex differences in spatial skill. Developmental Psychology, 35(4), pp. 940-949, 1999.

[8] Casey, M.B., et al., The influence of spatial ability on gender differences in mathematics college entrance test scores across diverse samples. Developmental Psychology, 31(4), pp. 497-705, 1995.

[9] Quaiser-Pohl, C. \& Lehmann, W., Girls' spatial abilities: Charting the contributions of experiences and attitudes in different academic groups. British Journal of Educational Psychology, 72(2), pp. 245 - 260, 2002.

[10] Pezaris, E. \& Casey, M.B., Girls who use "masculine" problem solving strategies on a spatial task: proposed genetic and environmental factors. Brain \& Cognition, 17, pp. 1-22, 1991.

[11] Thomsen, T., et al., Functional magnetic resonance imaging (fMRI) study of sex differences in a mental rotation task. Medical Science Monitor: International Medical Journal Of Experimental And Clinical Research, 6(6), pp. 1186-1196, 2000.

[12] Birenbaum, M., Kelly, A.E., \& Levi-Keren, M., Stimulus features and sex differences in mental rotation test performance. Intelligence, 19(1), pp. 51-64, 1994.

[13] Scali, R.M., Brownlow, S., \& Hicks, J.L., Gender differences in spatial task performance as a function of speed or accuracy orientation. Sex Roles - A Journal of Research, 43(5/6), pp. 359-376, 2000. 
[14] Sherman, J., Sex-related cognitive differences. Charles C. Thomas: Springfield, Illinois, 1978.

[15] Tracy, D.M., Toys, spatial ability, and science and mathematics achievement: Are they related? Sex Roles, 17(3/4), pp. 115-138, 1987.

[16] Mumtaz, S., Children's enjoyment and perception of computer use in the home and the school. Computers \& Education, 36, pp. 347-362, 2001.

[17] Baenninger, M.A. \& Newcombe, N., The role of experience in spatial test performance: A meta-analysis. Sex Roles, 20(5/6), pp. 327-344, 1989.

[18] Kass, S.J., Ahlers, R.H., \& Dugger, M., Eliminating gender differences through practice in an applied visual spatial task. Human Performance, 11(4), pp. 337-349, 1998.

[19] Okagaki, L. \& Frensch, P.A., Effects of video game playing on measures of spatial performance: gender effects in late adolescence, Interacting with video. ed. P.M. Greenfield \& R.R. Cocking, Ablex publishing corporation: Norwood, New Jersey, pp. 115-140, 1994.

[20] Lohman, D.F. \& Nichols, P.D., Training spatial abilities: Effects of practice on rotation and synthesis tasks. Learning and Individual Differences, 2(1), pp. 67-93, 1990.

[21] McClurg, P.A. \& Chaille, C., Computer games: environments for developing spatial cognition? Journal of educational computing research, 3(1), pp. 95-111, 1987.

[22] Subrahmanyam, K. \& Greenfield, P.M., Effect of video game practice on spatial skills in girls and boys, Interacting with video. ed. P.M. Greenfield \& R.R. Cocking, Ablex publishing corporation: Norwood, New Jersey, pp. 95-114, 1994.

[23] De Lisi, R. \& Cammarano, D.M., Computer experience and gender differences in undergraduate mental rotation performance. Computers in Human Behavior, 12(3), pp. 351-361, 1996.

[24] Stumpf, H. \& Klieme, E., Sex-related differences in spatial ability: more evidence for convergence. Perceptual \& Motor skills, 69(3), pp. 915-921, 1989.

[25] Cox, B. \& Thompson, S., Multiple bodies: Sportswomen, soccer and sexuality. International review for the sociology of sport, 35(1), pp. 5-20, 2000.

[26] McGuiness, D. \& Morley, C., Sex differences in the development of visuo-spatial ability in pre-school children. Journal of mental imagery, 15(3/4), pp. 143-150, 1991.

[27] Voyer, D., Voyer, S., \& Bryden, M.P., Magnitude of sex differences in spatial abilities: a meta-analysis and consideration of critical variables. Psychological Bulletin, 117(2), pp. 250-270, 1995.

[28] Kerns, K.A. \& Berenbaum, S.A., Sex differences in spatial ability in children. Behaviour Genetics, 21(4), pp. 383-396, 1991. 